\title{
Electroweak couplings of the $\tau$ lepton
}

\author{
JOHN SWAIN \\ Department of Physics, Northeastern University, Boston, MA 02115, USA
}

\begin{abstract}
The tau lepton is the heaviest known lepton and studies of its production and decay allow measurements of its couplings to the electroweak gauge bosons. In particular, sensitive tests of the idea of lepton universality are possible, with the hope that some light will be shed on not just the nature of the origin of mass, but also on why there seem to be three generations. The recent LEP results provide most of this experimental data, and very recently measurements of the anomalous magnetic and electric dipole moments of the tau have been performed. I present an overview of all these results, and discuss what this means in the framework of the Standard Model and attempts to go beyond it.
\end{abstract}

Keywords. Tau; electroweak; anomalous couplings.

PACS Nos 14.60.Fg; 13.35.Dx; 13.38.Dg; 13.40.Em

\section{Introduction}

While the search for the Higgs boson as the origin of mass is often regarded as the most pressing issue in particle physics today, a truly satisfactory theory of mass should be expected to give insight not only into how mass can be introduced consistently into a gauge theory based on $\mathrm{SU}(2)_{\mathrm{L}} \times \mathrm{U}(1)_{\mathrm{Y}}$, but also some idea of why masses are what they are, why there are three generations, and what the CKM mixing angles are. Indeed, the issue of mixing only arises in the presence of more than one generation of nonzero particle masses.

Given that the generation structure seems to group fermions together which differ only in mass and some obscure additive quantum number, it is interesting to see to what degree corresponding fermions are indeed similar. The only really accessible window on any potential breakdown of this structure is provided by the $\tau$ lepton. Electron-muon universality in the context of the Standard Model is well-established, but it is only recently that exhaustive studies of $\tau$ couplings have been possible. Of the other fermions one might study, the quarks are greatly complicated by the strong interaction, and the neutrinos by their stability and small (and indeed unknown) masses and tiny interaction cross sections.

\section{General couplings}

The results presented are grouped in the following way: we look first at non-derivative four-fermion couplings, then non-derivative gauge couplings, and finally derivative gauge couplings. 


\section{Non-derivative couplings}

\subsection{Charged current interactions}

Allowing a general combination of local scalar, pseudoscalar, vector, axial vector and tensor interactions, the 4-fermion interaction describing leptonic tau decays can be specified in terms of 4 Michel parameters $\rho, \eta, \xi, \xi \delta$ (expected to be 3/4, 0, 1, 3/4 for the Standard Model). Some knowledge of the $\tau$ spin direction is needed to obtain the Michel parameters. Taus are produced with intrinsic polarisation from $Z^{0}$ decays at LEP, but both at LEP and at lower energies an 'effectively polarized' sample can be obtained by noting that production of tau pairs from a $J=1$ state forces their spins to be highly correlated. Figure 1a shows the results [1] of fits to the observed tau leptonic decay spectra.

The best determination of $\eta$ is from total $\tau$ decay width [2] and yields $\eta_{\tau \mu}=0.009 \pm$ 0.022 . Measurements of the tau neutrino helicity from hadronic tau decays [1] are shown in figure $1 \mathrm{~b}$. Non-derivative couplings of the $\tau$ to on-shell $W$ bosons are also consistent with those of the $e$ and $\mu$ [3].

\subsection{Weak neutral current interactions}

Given the wealth of data from $Z^{0}$ decays to taus, we will concentrate here on the nonderivative gauge couplings to the $Z^{0}$ represented by a combination of vector and axial vector components. For discussions of 4-fermion contact interactions, see reference [4].

There are essentially four different measurements that can be contemplated in $e^{+} e^{-} \rightarrow$ $\tau^{+} \tau^{-}$: the total cross section, the forward-backward asymmetry, the average $\tau$ polarization, and the forward-backward asymmetry of the $\tau$ polarization. The latter two analyses

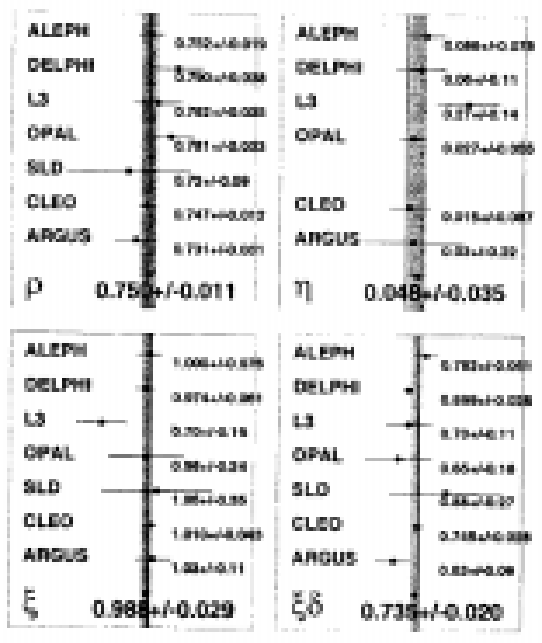

(a)

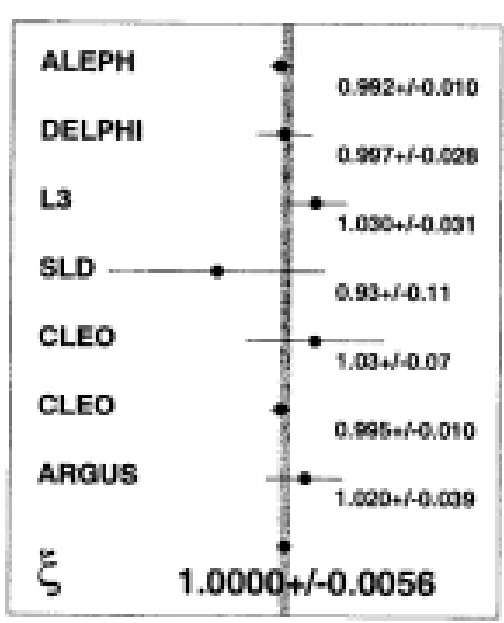

(b)

Figure 1. Nonderivative charged current couplings. (a) World average Michel parameters, following Stahl. (b) World average of tau neutrino helicity assuming $e-\mu$ universality, following Stahl. 


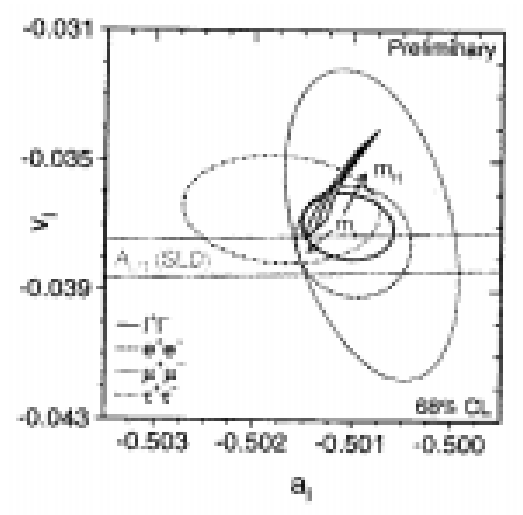

(a)

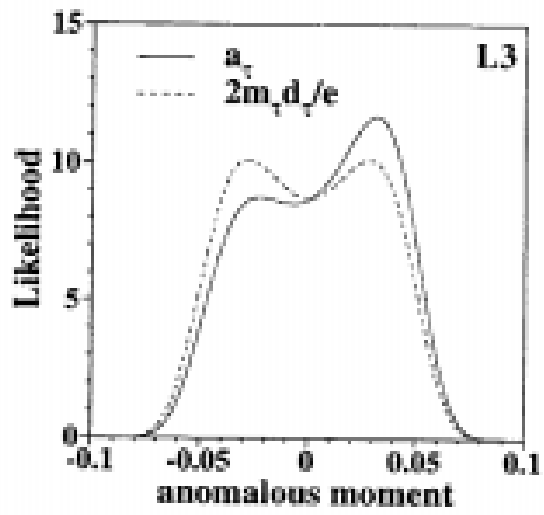

(b)

Figure 2. Fit results for Standard Model electroweak couplings and anomalous electromagnetic moments. (a) Vector and axial vector coupling constants of the $\tau$ to the $Z^{0}$. (b) Likelihood distributions for anomalous moment of the $\tau$ from the L3 analysis of $\tau \tau \gamma$ production.

are made possible by the fact that the weak charged current via which the $\tau$ decays has a handed $V-A$ structure so that the $\tau$ essentially acts as its own polarimeter.

A combination of all LEP electroweak data gives the results shown in figure $2 \mathrm{a}$ for the vector and axial vector coupling constants $v_{l}$ and $a_{l}$ to the $Z$ boson for the three charged leptons [3]. A band shows constraints from the left-right asymmetry measurement at SLD, and the dependence on the masses of the top quark and Higgs boson are also indicated.

\section{Derivative couplings}

Derivative couplings are excluded at tree-level in renormalizable lagrangians, but effective terms are expected due to loop diagrams in direct analogy to the slight shift of the $g$-factor from 2. Effective terms can also appear due to possible compositeness of the $\tau$.

\subsection{Anomalous electric and magnetic dipole moments of the $\tau$}

One can look for anomalous couplings of the $\tau$ lepton to the electromagnetic field by parametrizing the coupling of the $\tau$ lepton to photons of 4-momentum $q$, replacing the usual $\gamma_{\mu}$ coupling by

$$
\gamma_{\mu}+i \frac{F_{2}\left(q^{2}\right)}{2 m_{\tau}} \sigma_{\mu \nu} q^{\nu}-F_{3}\left(q^{2}\right) \sigma_{\mu \nu} q^{\nu} \gamma_{5} .
$$

This is the most general form allowed by Lorentz invariance neglecting a possible anapole moment term and assuming the $\tau$ has charge $e$ [5]. The form factors at $q^{2}=0$ and with the $\tau$ close to mass-shell have special significance : $F_{2}(0)=(g-2 / 2)=a_{\tau}$ anomalous magnetic moment of the tau, and $F_{3}(0)$ is the electric dipole moment $d_{\tau}$ of the tau. Hermiticity 
of the electromagnetic current forces all these form factors to be real, while $T$ invariance requires that $F_{3}=0$. Upper limits on $F_{2}\left(q^{2}\right)$ for $q^{2} \neq 0$ have been derived from their effect on the tau pair production cross section $[4,6]$.

The case of $q^{2}=0$ can be studied in radiative tau pair production $e^{+} e^{-} \rightarrow \tau^{+} \tau^{-} \gamma$. While $\tau$ 's at LEP are quite clean, the analysis of this data is highly nontrivial, requiring per mil accuracy on signal and background. A comparison of observed data [8] to a detailed theoretical calculation [9] of the expected spectrum including all interference and mass terms gives the likelihood distribution shown in figure 2b. If the $\tau$ mass is neglected [7], the large interference between the Standard Model and $F_{2}$ contributions is not included.

The corresponding constraints on anomalous moments from L3 are $a_{\tau}=0.004 \pm$ $0.027 \pm 0.023$ and $d_{\tau}=(0.0 \pm 1.5 \pm 1.3) \times 10^{-16} e \cdot \mathrm{cm}$.

A similar analysis from OPAL [10] using a simplified calculation of the expected effects of anomalous moments [11] gives $-0.068<a_{\tau}<0.065$ and $-3.8 \times 10^{-16} e \cdot \mathrm{cm}<d_{\tau}<$ $3.6 \times 10^{-16} e \cdot \mathrm{cm}$ at the $95 \%$ confidence level.

\subsection{Anomalous weak neutral electric and magnetic dipole moments}

By analogy with the electromagnetic case described above, we can add a term coupling the $\tau$ to the $Z^{0}$ gauge boson of the form

$$
\gamma_{\mu}+i \frac{e a_{\tau}^{W}}{2 m_{\tau}} \sigma_{\mu \nu} q^{\nu}-d_{\tau}^{W} \sigma_{\mu \nu} q^{\nu} \gamma_{5}
$$

to the Standard Model lagrangian and investigate the effects of the parameters $a_{\tau}^{W}$ and $d_{\tau}^{W}$ with tau pairs produced at the $Z^{0}$. The expectations for these quantities in the Standard Model are well below any chance of detectability with present data, but any large observed values would be a definite indication of new physics.

A number of variables can be constructed out of the 4-momenta of the $\tau$ decay products and the $\tau$ flight direction, and comparison with theoretical predictions yields LEP averages [13] of $\operatorname{Re}\left(d_{\tau}^{w}\right)\left|<3.0 \times 10^{-18} e \cdot \mathrm{cm}, \operatorname{Im}\left(d_{\tau}^{w}\right)\right|<9.2 \times 10^{-18} e \cdot \mathrm{cm}$, and $d_{\tau}^{w} \mid<9.4 \times$ $10^{-18} e \cdot \mathrm{cm}$.

For effects on single tau polarization, see [12]. L3 has the only limits [14] on $a_{\tau}^{W}$ : $\operatorname{Re}\left(a_{\tau}^{W}\right)=(0.0 \pm 1.6 \pm 2.3) \times 10^{-3}$ and $\operatorname{Im}\left(a_{\tau}^{W}\right)=(-1.0 \pm 3.6 \pm 4.3) \times 10^{-3}$.

\subsection{Anomalous charged electric and magnetic dipole moments}

Following Rizzo [15], and in direct analogy to what was done in the electromagnetic case, we can modify the Standard Model coupling of the $\tau$ to the $W$ by replacing $\gamma^{\mu}\left(1-\gamma_{5}\right)$ by

$$
\left(\gamma_{\mu}+\frac{i}{2 m_{\tau}} \sigma_{\mu \nu} q^{\nu}\left(\kappa-i \tilde{\kappa} \gamma_{5}\right)\right)\left(\gamma_{\mu}\left(1-\gamma_{5}\right)\right)
$$

The best results for these parameters are obtained from examination of their effect on the $\tau$ decay width [2] which yields: $\kappa=0.001 \pm 0.008$ and $\tilde{\kappa}=0.00 \pm 0.16$.

These are significantly better than previous results from branching ratios [15] $(|\kappa|<$ $0.0283)$ and an extension of Michel parameter analysis by DELPHI [16] $(\kappa=0.029 \pm$ $0.036 \pm 0.018)$. 


\section{Conclusions}

Having found that the $\tau$ does indeed seem to be just like an electron or muon aside from mass and its own mysterious lepton number, where does this leave us? Rather than despairing of failing to find a crack in the Standard Model, I suggest that the fact that universality holds so well despite repeated assaults is probably an important message about the structure of the universe, and may well turn out to be a valuable hint in our search for an understanding of the origin of mass.

\section{Acknowledgments}

I would like to thank the organizing committee for a very pleasant and well-organized conference, and all my collaborators on L3, especially in the tau analysis group. In particular I would like to thank M-T Dova, S Gau, T Paul, and L Taylor for fruitful collaborations on some of the topics discussed here, and A Stahl and A Zalite for useful discussions. This work was supported in part by the National Science Foundation in the United States and the International Center for Theoretical Physics in Trieste.

\section{References}

[1] A Stahl, 'Michel Parameters. Averages and Interpretations', Proceedings of TAU98, Nucl. Phys. vol. 76

[2] M-T Dova, J Swain and L Taylor, Phys. Rev. D58, 015005 (1998); Constraints on anomalous charged current couplings, tau neutrino mass and fourth generation mixing from tau leptonic branching fractions, Proceedings of TAU98, op. cit.

[3] The LEP electroweak working group, data for 1998 summer conferences, http://www.cern.ch/LEPEWWG/

[4] M C Gonzalez-Garcia, A Gusso, and F Novaes, eprint hep-ph/9802254

[5] W J Marciano, Proceedings BNL Summer Study on CP Violation edited by S Dawson and A Soni (Brookhaven National Laboratory, 1990)

[6] D J Silverman and G L Shaw, Phys. Rev. D27, 1196 (1983)

R Escribano and E Massó, Phys. Lett. B301, 419 (1993)

R Escribano and E Massó, Nucl. Phys. B429, 19 (1994)

G Köpp, D Schaile, M Spira, and P M Zerwas, Z. Phys. C65, 545 (1995)

[7] J A Grifols and A Méndez, Phys. Lett. B255, 611 (1991); erratum ibid B259, 512 (1991)

[8] L3 Collaboration: M Acciarri et al, Phys. Lett. B434, 169-179 (1998)

[9] S S Gau, T Paul, J Swain, and L Taylor, Nucl. Phys. B523, 439 (1998)

[10] OPAL Collaboration: K Ackerstaff et al, Phys. Lett. B431, 188 (1998)

[11] J Biebel and T Riemann, Z. Phys. C76, 53 (1997)

[12] J Vidal, J Bernabéu, and G González-Springberg, $\tau$ Weak Dipole Moments from Azimuthal Asymmetries, Proceedings of TAU98, op. cit.

[13] A Zalite, LEP Summary on Weak Dipole Moments of the $\tau$ Lepton, Proceedings of TAU98, op. cit.

[14] L3 Collaboration: M Acciarri et al, Phys. Lett. B426, 207 (1998)

[15] T G Rizzo, Phys. Rev. D56, 3074 (1997)

[16] P Seager, Michel parameters and limits on tensor couplings from DELPHI, Proceedings of TAU98, op. cit. 